\title{
Quadrupole Mode of the Sun's Poloidal Magnetic Field: on the Possibility of Exciting by Turbulent Dynamo Mechanism
}

\author{
V. N. Kryvodubskyj \\ Astronomical Observatory of Kyiv National University \\ Observatorna Street 3, Kyiv - 53, 04053, Ukraine
}

\begin{abstract}
On the base of turbulent dynamo model with allowance for newer helioseismic measurements of the Sun's inner rotation it is shown that the physical conditions in deep layers of the solar convection zone in near-pole domain are favourable for exciting of quadrupole mode of poloidal magnetic field near maximum phase of 23 -rd solar cycle
\end{abstract}

\section{Introduction}

Magnetographic observations of the poloidal field of the Sun evidence that this field may be generally described by simple magnetic dipole with antisymmetric polarity relatively to equator. In this case the synoptic maps of the polarity distribution of large-scale surface (backgraund) magnetic field manifaste the two latitudinal zones with opposed magnetic polarity in both sides from equator. However during last four maximums of the solar activity the both poles of the polar magnetic field had the same polarity. To interpret this observed magnetic anomaly some investigators considere that the quadrupole mode, which is symmetric relatively to equatorial plane, predominated in the poloidal field at these times. Then the maps of magnetic polarity distribution must display four latitudinal zones (two zones of opposite signs in each hemisphere). Here in the frame of the main magnetic field theory with allowance for newer heliosesmic measurements of the inner rotation rate we attemp to reveal the possibility of exciting of quadrupole mode of the poloidal field in the Sun's deep layers.

\section{2. turbulent dynamo model}

According to dynamo model the combination of differential rotation and helical (hyrotropic) turbulence in the solar convection zone (SCZ) near tachocline (a rigion of strong shear of angular velocity at the base of the SCZ) produces the toroidal and poloidal components of the Sun's global magnetic field $(\alpha \Omega$ dynamo mechanism). Parker (1955) has suggested to represent the solution of dynamo equations for global magnetic components in the form of migratory dynamo waves. Using the boundary conditions he brought the dynamo problem to solve the dispersion relations describing the character of the dynamo-effect. Excitation thresholds of multipole modes are determined by eigenvalues of the dispersion relations, i.e. numbers $K \lambda$ ( $K$ is the wave number of dynamo wave, $\lambda$ 
is the characteristic scale of the dynamo region). Parker (1971) found by model calculation that the exitation thresholds of the periodic dipole and quadrupole modes are rather close. Wave number is determined by physical parameters of the SCZ, $K=\left(\alpha G / \nu_{T}^{2}\right)^{1 / 3}$ ( $\alpha$ is the helicity parameter of turbulent convection, $G=r \partial \Omega / \partial r$ is the measure of the radial differential rotation $\partial \Omega / \partial r$ at the distance $r$ from the Sun's centre, $\nu_{T}$ is is the turbulent viscosity). The direction of dynamo-wave migration depends on a sign of the wave number. Magnetic wave travels equatorwards when $K \prec 0$, and polewards when $K \succ 0$.

Since the excitation thresholds of the periodic dipole and quadrupole modes are rather close then it is possible that the quadrupole will be excite for variations of physical parameters $\left(\alpha, \nu_{T}, \partial \Omega / \partial r\right)$ in a some regions of the SCZ. Recently helioseismic measurements (Tomczyk, Schou \& Thompson 1995; Howe et al. 2000) evidence that differential rotation strong depends on heliolatitude and depth in the SCZ. The inner regular velocity field is naturally devided on two domains of fast (near-equator domain 1: heliolatitudes $\theta=0^{\circ}-40^{\circ}$ ) and slow (near-pole domain 2: heliolatitudes $\theta=50^{\circ}-90^{\circ}$ ) rotation in each hemisphere, with opposite sign of $\partial \Omega / \partial r$ in each domain. The angular velocity decreases with depth $(\partial \Omega / \partial r \succ 0)$ in the near-equator domain, while it increases $(\partial \Omega / \partial r \prec 0)$ in the near-pole domain. We calculated numbers $K \lambda$ near tachocline (on the depth $175,000 \mathrm{~km}, r \approx 5.2 \cdot 10^{10} \mathrm{~cm}$ ) for the SCZ model by Stix (1989) using our estimation of the helicity parameter (Krivodubskij 1998) and the values of the radial gradient of angular velocity obtained from newer helioseismic measurements near maximum phase of 23-rd solar cycle (May 1995 - November 1999) (Howe et al. 2000). It was found the calculated value $K_{1} \lambda_{1}$ to be about -7 in domain $1\left(\lambda_{1} \approx 2 \pi r\left(40^{\circ} / 360^{\circ}\right) \approx 3.6 \cdot 10^{10} \mathrm{~cm}\right)$ at the latitude $20^{\circ}$ (where $\alpha_{1} \approx-2 \cdot 10^{2}$ $\mathrm{cm} / \mathrm{s}, \partial \Omega^{(1)} / \partial r \approx+6.3 \cdot 10^{-18} \mathrm{rad} / \mathrm{s} \cdot \mathrm{cm}, G_{1}=r \partial \Omega^{(1)} / \partial r \approx+3.3 \cdot 10^{-7} \mathrm{rad} / \mathrm{s}$, $\left.\nu_{T} \approx 3 \cdot 10^{12} \mathrm{~cm}^{2} / \mathrm{s}\right)$. The value obtained is close to the eigenvalue of -7.43 by Parker (1971) which is necessary for dipole exciting. The radial gradient velocity changes its sign on opposite at the latitides higher than $50^{\circ}$ so the value $K_{2} \lambda_{2}$ also changes its sign in the domain $2\left(\lambda_{2} \approx 3.6 \cdot 10^{10} \mathrm{~cm}\right)$ at the latitude $70^{\circ}\left(\alpha_{2} \approx-3 \cdot 10^{2} \mathrm{~cm} / \mathrm{s}, \partial \Omega^{(2)} / \partial r \approx-8 \cdot 10^{-18} \mathrm{rad} / \mathrm{s} \cdot \mathrm{cm}, G_{2}=r \partial \Omega^{(2)} / \partial r\right.$ $\left.\approx-4 \cdot 10^{-7} \mathrm{rad} / \mathrm{s}, \nu_{T} \approx 3 \cdot 10^{12} \mathrm{~cm}^{2} / \mathrm{s}\right)$. Here $K_{2} \lambda_{2}$ amounts to $\approx+8$ that is near to eigenvalue +7.43 , necessary for quadrupole exciting.

Thus, near maximum phase of 23-rd solar cycle the turbulent dynamo mechanism in near-equator domain 1 in vicinity of the tachocline produces the dipole mode of the poloidal field (migrated equatorwards since $K_{1} \prec 0$ ); while in nearpole domain 2 the conditions are more favourable for exciting the quadrupole mode (migrated polewards, $K_{2} \succ 0$ ). As a result, the maps of polarity distributions of the observed large-scale photospheric magnetic field in the northern hemisphere display three latitudinal zones with intermittent polarity, and two latitudinal zones in the southern hemisphere. In this case the both near-pole zones must have the same magnetic signs. This gives possibility to explain the surface polar magnetic symmetry relatively to equator at solar maximum.

\section{References}

Howe, R., Christensen-Dalsgaard, J., Hill, F., Komm, W., Larsen, R.M., Schou, J., Thompson, M.R., Toomre, J. 2000, Science, 287, 2456. 
Krivodubskii, V.N. 1998, Azh, 75, 139.

Parker, E.N. 1955, Apj, 122, 293.

Parker, E.N. 1971, Apj, 164, 491.

Stix, M. 1989, The Sun, (Berlin: Verlag), 200.

Tomczyk, S., Schou, J., Thompson, M.J. 1995, Apj, 448, L57. 